\title{
Uniaxial stress study of the transport properties of the quasi-two-dimensional organic superconductor $(\mathrm{BEDT}-\mathrm{TTF})_{2} \mathrm{Cu}(\mathrm{NCS})_{2}$
}

\author{
C.E. Campos ${ }^{a}, *$, J.S. Brooks ${ }^{a}$, P.J.M. van Bentum ${ }^{\text {, J.A.A.J. Perenboom }}{ }^{\text {, }}$, S.J. Klepper ${ }^{\mathrm{c}}$,

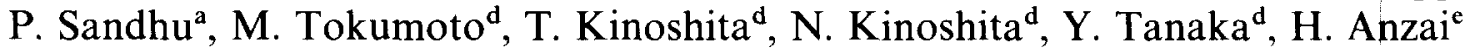 \\ ${ }^{2}$ Department of Physics, Boston University. 590 Commonwealth Ave., Boston, MA 02215, USA \\ ${ }^{b}$ High Field Magnet Laboratory and Research Institute for Materials, University of Nijmegen, NL-6525 ED Nijmegen, The Netherlands \\ ${ }^{\mathrm{c}}$ F.B. National Magnet Laboratory, Massachusetts Institute of Technology, Cambridge, MA 02139, USA \\ ¿Electrotechnical Laboratories, Tsukuba, Ibaraki 305, Japan \\ ${ }^{\mathrm{e}}$ Himeji Institute of Technology, 2167 Shosya. Himeji, Hyogo 671-22, Japan
}

\begin{abstract}
We report the effects of uniaxial stress applied along the least conducting direction ( $a$-axis) of the organic superconductor $\kappa-(\mathrm{BEDT}-\mathrm{TTF})_{2} \mathrm{Cu}(\mathrm{NCS})_{2}$. We find that the superconducting transition temperature decreases with stress $\left(\mathrm{d} T_{\mathrm{e}} / \mathrm{d} p_{\mathrm{a}}=-2 \mathrm{~K} / \mathrm{kbar}\right)$. Furthermore, we find an increase in the area of the closed orbits on the Fermi surface with stress. These observations are at variance with predictions based solely on the expected expansion of the conducting plane unit cell by the Poisson effect. We discuss our results in the light of previous stress measurements.
\end{abstract}

\section{Introduction}

$\kappa-(\mathrm{BEDT}-\mathrm{TTF})_{2} \mathrm{Cu}(\mathrm{NCS})_{2}$ has been one of the most studied compounds of the (BEDT-TTF) ${ }_{2} \mathrm{X}$ (where $\mathrm{X}$ is one of a number of anion structures) class of quasi-twodimensional organic conductors [1] due to its large transition temperature $\left(T_{\mathrm{c}}=11 \mathrm{~K}\right)$ and correspondingly large critical field anisotropy. It also has the highest hydrostatic pressure derivative of $T_{\mathrm{c}}$ of any superconducting material, with values reported to be as high as $\mathrm{d} T_{\mathrm{c}} / \mathrm{d} p=-3 \mathrm{~K} / \mathrm{kbar}[2,3]$. This rapid decrease of $T_{\mathrm{c}}$ may arise from several factors. First, $T_{\mathrm{c}}$ is proportional to the density of states at the Fermi energy, which is in turn inversely proportional to the width of the energy band. In the tight-binding model used for band structure calcu-

* Corresponding author. lations in these materials [1], the width of the energy band is directly proportional to the density of states at the Fermi energy, which is in turn inversely proportional to the transfer integral between the BEDT-TTF molecules, which is increased when the molecules in the planes are compressed. Hence the decrease of $T_{\mathrm{c}}$ with hydrostatic pressure. A second reason for a decrease in $T_{\mathrm{c}}$ may arise from a strong pressure dependence of the effective mass, which decreases accordingly with $T_{\mathrm{c}}$. This has been observed in both the title compound [3] and also in the organic superconductor $\alpha$-(BEDT-TTF $)_{2} \mathrm{NH}_{4} \mathrm{Hg}(\mathrm{SCN})_{4}$ [4].

Because of the high anisotropy of the material, uniaxial stress methods are important to further understand the nature of the superconducting properties. There have been three previous studies. Kusuhara et al. [5] found $T_{c}$ to increase with tensile stress applied along the $b$-axis direction. Their method consisted of fixing two ends of 
a single crystal of $\kappa-(\mathrm{BEDT}-\mathrm{TTF})_{2} \mathrm{Cu}(\mathrm{NCS})_{2}$ to a substrate (copper or fused quartz) and use the differential thermal contraction between the crystal and the substrate to, upon cooling of the assembly, apply tensile stress in the $b$-axis direction - the short axis along the BEDT-TTF planes [6]. The increase in $T_{\mathrm{c}}$ varied between 0.5 and $2 \mathrm{~K}$, depending on the crystal and the type of substrate used. However, when the stress was applied to the molecular planes by sticking the crystal plane to the substrate, $T_{\mathrm{c}}$ decreased slightly. Tokumoto et al. [7] also found $T_{\mathrm{c}}$ to decrease when low (up to $10 \mathrm{bar}$ ) uniaxial compressive stress was applied along the $a$-axis, the crystal direction perpendicular to the molecular planes. Lang et al. [8] and Kunda et al. [9] have extracted the $T_{\mathrm{c}}$ stress derivatives for all crystal directions from the discontinuities in the thermal expansion coefficients and a comparison with the hydrostatic pressure derivative. Both groups obtained negative derivatives for stress along the $a$-axis, albeit with somewhat different absolute values. For stress along the $b$ or $c$-axes the signs of the derivatives reported by one group were opposite to those found by the other. In the present work described below, we have directly applied uniaxial stress along the $a$-axis, and report both the stress dependence of $T_{\mathrm{c}}$ and the stress dependence of the Shubnikov-de Haas oscillations.

\section{Experimental}

We have devised a sample preparation technique that allows the application of high (up to 3 kbar) uniaxial compressive stress along the $a$-axis to these fragile crystals, and have used it to measure the stress dependence of both $T_{\mathrm{c}}$ and the magnetoresistance (MR) of $\mathrm{K}$-(BEDT$\mathrm{TTF})_{2} \mathrm{Cu}(\mathrm{NCS})_{2}$. The basic idea is to compensate for the crystal's fragility and surface irregularities by encapsulating it in a medium with closely matched physical properties and whose shape can be optimized to sustain the applied stress. Four gold wires were bonded with gold paint to the plate-like faces of a small $\left(\approx 0.5 \times 0.5 \mathrm{~mm}^{2}\right.$ faces) and thin $(\leq 0.2 \mathrm{~mm})$ single crystal of $\kappa$ (BEDT-TTF $)_{2} \mathrm{Cu}(\mathrm{NCS})_{2}$ for the four terminal resistance measurement across the BEDT-TTF planes. The crystal was placed in a drop of liquid two-component epoxy between two sheets of kapton with its flat faces parallel to the sheets. A small, solid cylindrical crystal-epoxy tube was obtained after the epoxy cured. Stress was then applied on the flat faces of the tube, that is, along the crystal's $a$-axis, using a pneumatic piston in a ${ }^{3} \mathrm{He}$ insert. A more detailed description of the sample preparation method and the stress apparatus can be found in Ref. [10]. The resistance was measured by AC lock-in detection $(f=17.77 \mathrm{~Hz})$ with currents parallel to the $a$-axis in the range $10-100 \mu \mathrm{A}$. Magnetoresistance measurements were done in the resistive magnets at the Francis Bitter National Magnet Laboratory.

\section{Results}

The $a$-axis uniaxial stress dependence of the resistance of $\kappa$-(BEDT-TTF $)_{2} \mathrm{Cu}(\mathrm{NCS})_{2}$ at low temperatures is shown in Fig. 1. As the stress increases, the normal state resistivity is reduced and the transition to the superconducting state moves from $11 \mathrm{~K}$ to lower temperatures. This value of $T_{\mathrm{c}}$ at zero stress is identical to that measured on bare crystals from the same batch, but the width of the superconducting transition increases from $2 \mathrm{~K}$ on the bare crystals to $4 \mathrm{~K}$ for the one cooled in epoxy (see Fig. 1 inset). This last fact indicates that stress inhomogeneity can be induced in these very thin samples during the initial epoxy cure and/or during cool down. However, both the onset and the full transition points decrease linearly at a rate of $\mathrm{d} T_{\mathrm{c}} / \mathrm{d} p_{\mathrm{a}}=-2 \mathrm{~K} / \mathrm{kbar}$, which indicates that the inhomogeneity present initially is not increased appreciably when stress is applied to the sample. Moreover, the applied stress was fully reversible

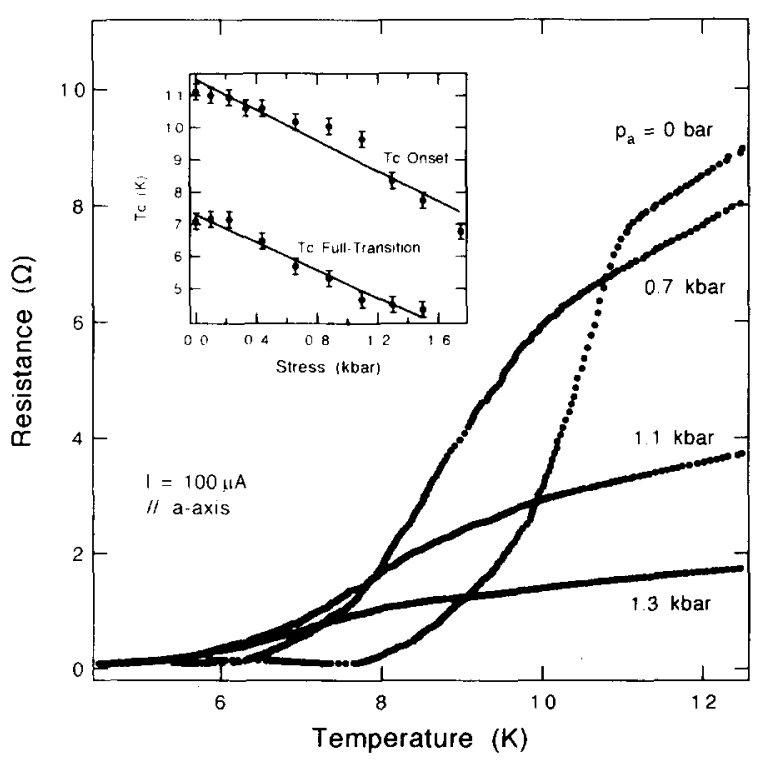

Fig. 1. Resistance of $\kappa$-(BEDT-TTF $)_{2} \mathrm{Cu}(\mathrm{NCS})_{2}$ around the transition temperature for different values of $a$-axis stress. Inset: superconducting transition temperature variation with stress. Slopes of the lines fitted to the onset and full-transition temperatures are -2.1 and $-2.0 \mathrm{~K} / \mathrm{kbar}$, respectively. (The onset temperature of the superconducting transition was taken to be the point where the normal state resistance slope first changes and the fill-transition temperature was assigned to the point where the slope becomes zero.) 
in the range measured, with $T_{\mathrm{c}}$ returning to the previous higher values as pressure in the piston was released. The decrease of $T_{\mathrm{c}}$ with stress applied along the $a$-axis agrees with previous reports [7-9], although higher rates of $\mathrm{d} T_{\mathrm{c}} / \mathrm{d} p_{\mathrm{a}}=-4.8 \mathrm{~K} / \mathrm{kbar}[8]$ and $-3.2 \mathrm{~K} / \mathrm{kbar}$ [9] were extracted from the discontinuity in the thermal expansion coefficient.

In order to better understand the changes caused by uniaxial stress to the superconducting state, we also measured the MR of two different crystals of this compound at $0.55 \mathrm{~K}$. These preliminary measurements were restricted by the fact that these two crystals could not support stress above $1 \mathrm{kbar}$. However, similar MR features for stress below $1 \mathrm{kbar}$ (Fig. 2) were observed for both crystals. The normal state MR decreases and flattens with the applied stress and the critical field $\left(B_{\mathrm{c} 2}\right)$ moves to lower field values. The decrease of $B_{\mathrm{c} 2}$ accompanies the decrease of $T_{\mathrm{c}}$ found for the first sample and is linear in this stress range (Fig. 3). At 0 kbar (no gas pressure on the piston) the frequency of the Shubnikovde Haas $(\mathbf{S d H})$ oscillations, clearly seen superposed on the background MR, is $654 \mathrm{~T}$. This value falls within the wide range of reported atmospheric pressure frequencies (596-667 T [11]), and corresponds to the closed hole orbits on the quasi-two-dimensional Fermi surface [12]. It is possible that slight sample misalignment with respect to the magnetic field leads to the higher frequencies measured here and in a few other reports [11]. By $0.9 \mathrm{kbar}$ the amplitude of the SdH oscillations has been reduced, an indication that the stress on the sample is not completely homogoneous [10]. Nevertheless, the fundamental SdH frequency peak is still clearly visible on the Fourier spectrum, and is shifted to a higher value of $672 \mathrm{~T}$.

\section{Discussion}

If one considers the Poisson effect, stress applied along the crystal direction should expand the unit cell in the $b-c$ plane. This in turn decreases the size of the Brillouin zone, and all topological entities within it. Therefore the extremal orbits and the corresponding $\mathrm{SdH}$ frequencies should decrease, as has been observed in $\alpha$ (BEDT-TTF $)_{2} \mathrm{KHg}(\mathrm{SCN})_{4}$ [13]. As mentioned in the introduction, an expansion of the unit cell should give an increase in the density of states which would result in an increase in $T_{\mathrm{c}}$. Clearly neither of these expectations is realized for $\mathrm{K}$-(BEDT-TTF $)_{2} \mathrm{Cu}(\mathrm{NCS})_{2}$

There are several possible origins for the observed behavior. The first thing to bear in mind is that, since the stress is applied in the least conducting direction, the $a$-axis bandwidth increases and drives the system more three-dimensional. The difference in the anion layer

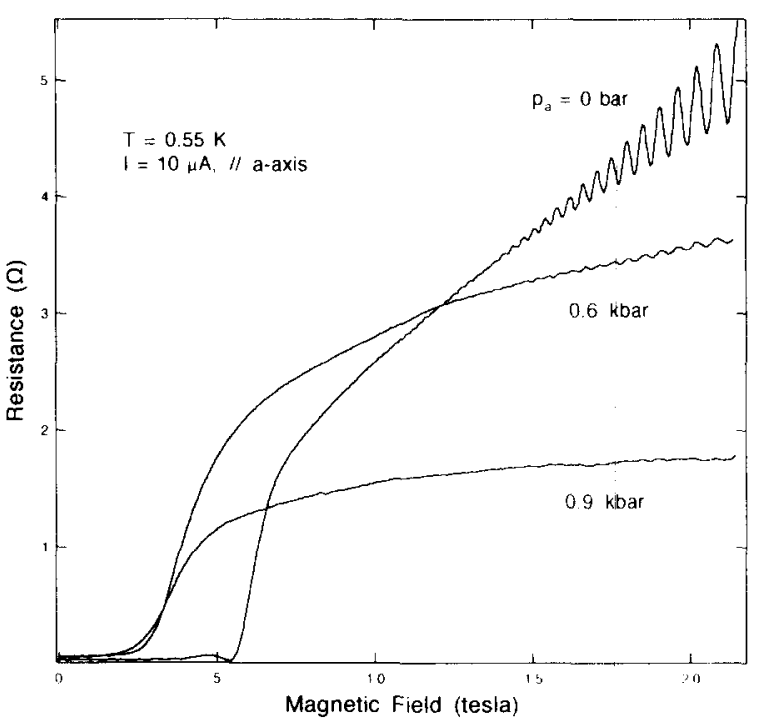

Fig. 2. Magnetoresistance of $\kappa-(\mathrm{BEDT}-\mathrm{TTF})_{2} \mathrm{Cu}(\mathrm{NCS})_{2}$ as a function of $a$-axis stress.

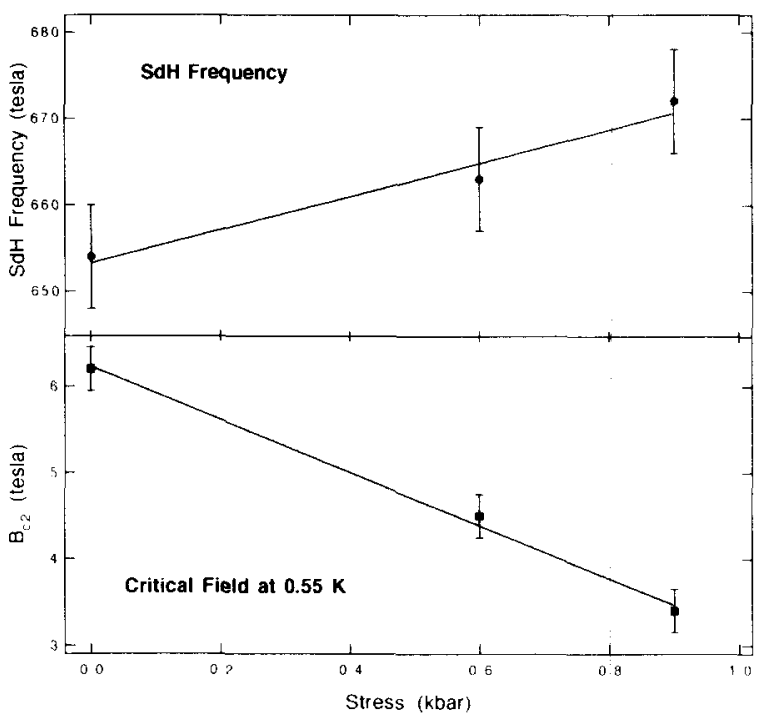

Fig. 3. Shubnikov-de Haas oscillation frequency (top graph) and upper critical field at $0.55 \mathrm{~K}$ (bottom graph) versus stress. Dotted lines in graphs are guide to the eyes. (The upper critical field was taken to be the middle resistance point at the transition between the zero and the linear magnetoresistance regimes.)

thickness between $\alpha$-(BEDT-TTF ${ }_{2} \mathrm{KHg}(\mathrm{SCN})_{4}$ and $\kappa$ $(\mathrm{BEDT}-\mathrm{TTF})_{2} \mathrm{Cu}(\mathrm{NCS})_{2}$, which are 7 and $3 \AA$ respectively, indicates that inter-layer interactions can be strong in the latter salt. However, the case is not so simple because the stress derivatives of $T_{\mathrm{c}}$ along the two 
$b-c$ plane directions have been found to have opposite signs [5], even though both stresses would be accompanied with a reduction in the inter-layer separation. Therefore there could exist some significant non-linearities in the adjustments of the positions of the molecules that, depending on the direction along which the stress is applied, might lead to an increase or a decrease in the energy band width. A second possible explanation is that in $\mathrm{K}$-(BEDT-TTF $)_{2} \mathrm{Cu}(\mathrm{NCS})_{2}$ there is a negative Poisson effect due to the nature of the until cell structure: the BEDT-TTF molecules in this salt are dimerized in an alternating square pattern [6], whereas in $\alpha$ (BEDT-TTF $)_{2} \mathrm{KHg}(\mathrm{SCN})_{4}$ they are arranged in a chevron-type staking with alternating rows. A negative Poisson effect would reconcile both the behavior of $T_{\mathrm{c}}$ and the $\mathrm{SdH}$ frequency with stress. As a third possibility, we note that since the electron-electron and the electron-phonon interactions appears to by pressure dependent, this may by itself drive $T_{\mathrm{c}}$ down irrespective of geometrical factors $[3,4]$. Finally, it is becoming evident that the band structure in these materials is very dependent on the details of the molecular overlap integrals [14]. Hence there could be significant changes in the Fermi surface topology which do not follow the simple reduced Brillouin zone model.

\section{Conclusions}

The present work has produced some surprises in the stress dependent properties of this highly anisotropic organic superconductor. Questions about the band structure, the mechanism of superconductivity, and the role of the inter-layer interaction (which is clearly changing by the nature of the experiment) have been raised. Further work, including stress studies along different axes by our new technique, is clearly in order.

\section{Acknowledgements}

Work at Boston University is supported by NSFDMR-92-14889. C.E.C. is supported by a fellowship from Junta Nacional de Investigação Cinetifica e Tecnológica and acknowledges the hospitality of the staff of the High
Field Magnet Laboratory where the stress method was initially tested.

\section{References}

[1] For a recent review of this subject see T. Ishiguro and K. Yamaji, Organic Superconductors (Springer, Berlin, 1990); J.M. Williams, J.R. Ferraro, R.J. Thorn, K.D. Carlson, U. Geiser, H.H. Wang, A.M. Kini and M. Whangbo, Organic Superconductors (Including Fullerenes) (Prentice-Hall, Englewood Cliffs, NJ, 1992); see also P.A. Mansky, P.M. Chaikin and R.C. Haddon, Phys. Rev. Lett. 70 (1993) 1323.

[2] J.E. Schirber, E.L. Venturini, A.M. Kini, H.H. Wang, J.R. Whiworth and J.M. Williams, Physica C. 152 (1988) 157.

[3] J. Caulfield, W. Lubczynski, F.L. Pratt, J. Singleton, D.Y.K. Ko, W. Hayes, M. Kurmoo and P. Day, J. Phys.: Condens. Matter 6 (1994) 2911.

[4] X. Chen, PhD Thesis, Boston University (1993); X. Chen et al., to be published.

[5] H. Kusuhara, Y. Sakata, Y. Ueba, K. Tada, M. Kaji and T. Ishiguro, Solid State Commun. 74 (1990) 251.

[6] H. Urayama, H. Yamochi, G. Saito, S. Saito, A. Kawamoto, J. Tanaka, T. Mori, Y. Maruyama and H. Inokuchi, Chem. Lett. (1988) 55.

[7] M. Tokumoto, K. Murata, N. Kinoshita, K. Yamaji, H. Anzai, Y. Tanaka, Y. Hayakawa, K. Nagasaka and Y. Sugawara, Mol. Cryst. Liq. Cryst. 181 (1990) 295.

[8] M. Lang, R. Modler, F. Steglich, N. Toyota and T. Sasaki, Physica B 194-196 (1994) 2005.

[9] M. Kund, J. Lehrke, W. Biberacher, A. Lerf and K. Andres, in: Int. Conf. on Science and Technology of Synthetic Metals (ICSM 94), Seoul, Korea (1994), submitted.

[10] C.E. Campos, J.S. Brooks, P.J.M. van Bentum, J.A.A.J. Perenboom, J. Rook, S.J. Klepper and M. Tokumoto, Rev. Sci. Instrum., to be published.

[11] See Refs. [3, 12] and J. Wosnitza, G.W. Crabtree, H.H. Wang, U. Geiser, J.M. Williams and K.D. Carlson, Phys. Rev. B 45 (1992) 3018 and references therein.

[12] K. Oshima, T. Mori, H. Inokuchi, H. Urayama, H. Yamochi and G. Saito, Phys. Rev. B 38 (1988) 938.

[13] C.E. Campos, J.S. Brooks, P.J.M. van Bentum, J.A.A.J. Perenboom, S.J. Klepper, P. Sandhu, M. Tokumoto, T. Kinoshita, N. Kinoshita, Y. Tanaka and H. Anzai, in: Int. Conf. on Science and Technology of Synthetic Metals (ICSM 94), Seoul, Korea (1994), submitted.

[14] L. Duscasse and A. Fritsch, Solid State Commun. 91 (1994) 201. 